\title{
Reducing Infectious Complications after Transrectal Prostate Needle Biopsy Using a Disposable Needle Guide: Is It Possible?
}

\author{
Cenk Gurbuz, Lutfi Canat, Gokhan Atis, Turhan Caskurlu
}

Istanbul Goztepe Training Hospital, Department of 2nd Urology, Istanbul, Turkey

\begin{abstract}
Purpose: To investigate whether the use of a disposable needle guide results in a decreased incidence of infectious complication after transrectal prostate needle biopsy (TPNB).

Materials and Methods: Fifty five patients who underwent 10-core TPNB were randomized into two groups. A pre-biopsy blood and urine examination was performed in both groups. Group 1 (25 patients) underwent biopsy with disposable biopsy needle guide and Group 2 (30 patients) underwent biopsy with reusable biopsy needle guide. All patients had a blood and negative urine culture before the procedure. The patients received ciprofloxacin $500 \mathrm{mg}$ twice a day beginning the day before the biopsy and continued for 3 days after. Serum C-reactive protein levels and urine and blood specimens were obtained $48 \mathrm{~h}$ after the biopsy. Primary endpoint of the study was to determine the effect of needle guide on the bacteriologic urinary tract infection (UTI) rate and secondary end point was to determine symptomatic UTI.

Results: The mean age of the patients was 63.46 (range 55 to 68) years. There were no significant differences regarding the prostate-specific antigen level, prostate size, existence of comorbidity in two groups before the procedure. Bacteriologic and symptomatic UTI was detected in $4 \%$ vs. $6.6 \%$ and $4 \%$ vs. $3.9 \%$ in Group 1 and 2 relatively $(\mathrm{P}>0.05)$.

Conclusion: The use of a disposable needle guide does not appear to minimize infection risk after TPNB. Large scale and randomized studies are necessary to determine the effect of disposable needle guide on infection rate after TPNB.
\end{abstract}

Key words: prostate; biopsy; needle; infection

Int Braz J Urol. 2011; 37: 79-86

\section{INTRODUCTION}

Transrectal ultrasound (TRUS) guided needle biopsy of the prostate is the most common modality used to diagnose prostate cancer. Since male individuals in a screening population are asymptomatic, biopsy procedure should be safe and morbidity should be kept to a minimum. Transrectal prostate needle biopsy (TPNB) is safe for diagnosing prostate cancer with few major, but frequent minor complications, such as hematuria and hematospermia $(1,2)$. Infectious complications after TPNB are an important issue of concern. Currently, many urologists use prophylactic antibiotic therapy and/or enemas to minimize infection-related complications; however, such therapy does not completely eliminate the risk of infection (3). The infectious complications associated with this procedure include: asymptomatic bacteriuria, fever, symptomatic urinary tract infections (UTIs), and bacteremia $(4,5)$.

Although many biopsy protocols have been described to reduce infectious complications after a 
TPNB, such as modified bowel preparation, different type and duration of antibiotic prophylactic schemes, and number of biopsy cores $(6,7)$. The role of a disposable needle guide to reduce the infection rate has not been adequately assessed. The notion that biopsy equipment, such as reusable guides, may be important as a contamination site for bacteremia, was first evaluated by Tuncel et al. (8). A disposable TRUS-probe needle guide (Matek; Geotek, Inc., Ankara, Turkey) has been developed to reduce the possible source of infectious complications and has been on the market in Turkey for 3 years.

We prospectively investigated whether the novel disposable TRUS-probe needle guide may decrease infectious complications after TPNB.

\section{MATERIAL AND METHODS}

Between January and June 2009, 55 patients were included in this study. All participants gave written informed consent. The local ethics committee approved the study. The mean age of the patients was 63.46 years (range 58-66 years) in the present study. The patients in whom TRUS-prostate biopsy was planned because of elevated prostate-specific antigen (PSA) levels were included in the study. Exclusion criteria included the following: patients who had an indwelling Foley catheter, symptomatic or asymptomatic UTIs, bleeding disorders, acute prostatitis before prostate biopsy, a previous prostatic biopsy or prostatic surgery, or any antibiotic treatment or anticoagulant therapy.

A midstream urine culture, serum $\mathrm{C}$-reactive protein (CRP) level, and WBC count of all patients were obtained two days before the procedure. The biopsy procedure was performed with the patients in the left lateral decubitus position using a Toshiba Fabio scanner with a $7.5 \mathrm{MHz}$ bi-planar probe attached(Toshiba, Tokyo, Japan).

Patients were randomized into 2 groups by using sealed, opaque envelopes; in Group 1 (25 patients) the biopsy was performed with disposable TRUS-probe needle guide; and in Group 2 (30 patients) the biopsies were performed with a reusable needle guide. A completely disposable apparatus of single unit construction designed for operative use in conjunction with a transrectal ultrasound probe when performing TPNB are presented in Figure-1. The separable and detachable features of the reusable needle guide are presented in Figure- 2 .

Peri-operative prophylaxis included administration of $500 \mathrm{mg}$ of ciprofloxacin orally the night before the procedure, and an enema per rectum 1 hour before the procedure. Patients were instructed to continue to take ciprofloxacin $500 \mathrm{mg}$ p.o. bid 3 days after the procedure.

Before the biopsy, a standard condom was fitted over the distal tip of the TRUS-probe and filled with lubricant gel. Depending upon randomization, either a reusable or a disposable needle guide was then fitted over the probe and the first condom. A second condom was fitted over these items and filled with lubricant gel. All patients received local anesthesia, consisting of $10 \mathrm{~mL} \mathrm{1 \%} \mathrm{lidocaine.} \mathrm{Injection} \mathrm{was}$ performed around the neurovascular bundle at the prostatic base and apical location with an 18 gauge 20 $\mathrm{cm}$ needle by inserting the needle through the needle guide under TRUS guidance and entering Denonvilliers' fascia. Infiltration was done on each side of the

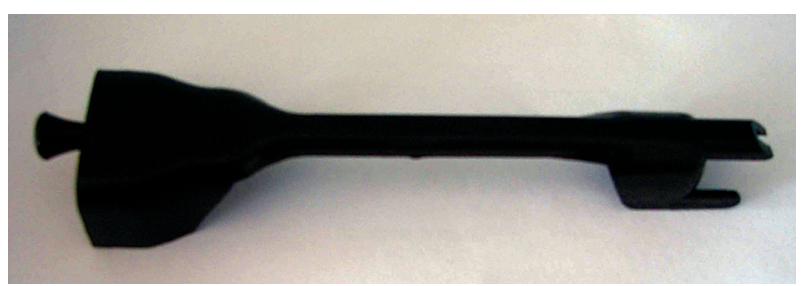

Figure1 - Disposable Needle Guide (Matek; Geotek, Inc., Ankara, Turkey) disposable needle guide.

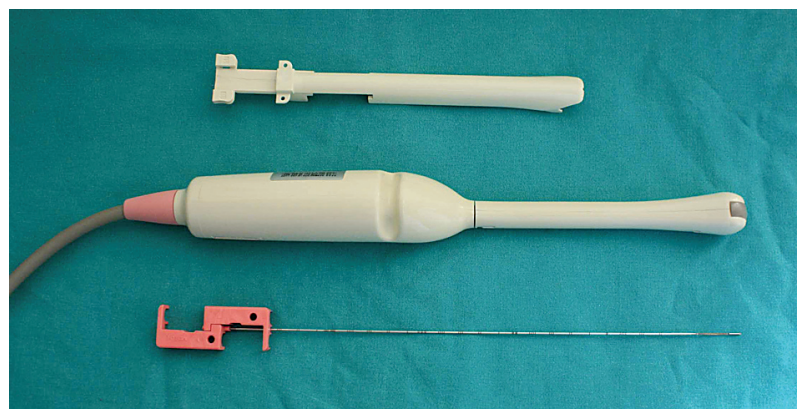

Figure 2 - Reusable needle guide and transrectal probe. 
prostate for a total of 2 punctures. The 10 core prostate biopsies were taken with a spring-loaded biopsy gun (C.R. Bard, Inc., Covington, GA, USA) and an 18 gauge Tru-cut biopsy needle. The core sampling notch was approximately $17 \mathrm{~mm}$ long by $1.2 \mathrm{~mm}$ in diameter. Repeated biopsy specimen collections were performed using the same needle. The procedure including ultrasound examination and biopsy required 10 minutes on average.

Upon completion of the procedure, the ultrasound probe was disinfected by wiping it with a $3.2 \%$ glutaraldehyde solution. The reusable needle guide has two separate pieces which form a lumen after being attached together. The reusable needle guide was soaked in a glutaraldehyde solution for at least 30 minutes, then rinsed with sterile saline and wiped off.

UTI was defined as bacteriologic UTI (bUTI): A patient with a blood or urine culture positive for a known uropathogen and symptomatic UTI (sUTI): A patient with an acute onset of one or more symptoms of dysuria, hematuria, frequency, urgency, urinary retention, suprapubic pain, flank pain, or rigors that have positive urine culture (a growth of $\geq 10^{5}$ colony forming units per $\mathrm{mL}$ ).

A midstream urine culture and blood cultures were obtained from each patient on day 2 after the biopsy and repeated on day 14 after the biopsy. Patients recorded oral body temperature twice daily for 2 weeks. Patients were asked to return for a follow-up visit 14 days after the biopsy. They were also advised to return immediately if they had any complication, such as a high fever, chills.

Primary end-point of the study was to determine the effect of needle guide on the bUTI rate. Secondary end point was sUTI. Clinical symptoms and signs of infection were monitored weekly by office interview.

Complications necessitating hospitalization during the 2 week post-biopsy period were defined as serious. Statistical analysis was performed using the Statistical Package for Social Sciences (SPSS) for Windows Version 13.0 software (SPSS, Inc, Chicago, IL, USA). Mean ages, serum total PSA levels, total prostate volume, comorbidities, CRP and WBC in the groups were compared using univariate analysis of variance test. Chi-squared test was used to compare infectious complication rates between the groups. A $\mathrm{p}$ value less than 0.05 was considered significant with a $95 \%$ confidence interval.

\section{RESULTS}

There were no significant differences between the two groups before the TPNB with respect to PSA levels, co-morbidities, CRP levels, and WBC counts. The data for each group is summarized in Table-1. The infectious complications are presented in Table2 . Bacteriologic UTI was observed in 3 patients ( 1 in Group 1 and 2 in Group 2). A total of 2 patients had Escherichia Coli (E. Coli) in blood culture (1 in each Group) and CRP levels were four times higher than before the TPNB in both groups. Two patients (1 in each Group) with high fever were admitted to the hospital and received intravenous antibiotic therapy. After receiving intravenous antibiotic therapy, the high fever and elevated CRP levels were controlled within 48 hours. E. Coli was harvested in urine culture in 1 patient in Group 2. This patient required hospitalization due to extended-spectrum beta-lactamase (ESBL) producing E. Coli harvested in urine culture in Group 2. No elevated CRP level or any signs of infection were recorded in this patient. Symptomatic UTI was diagnosed in 2 patients (1 patient in each group) caused by E. coli and received oral antibiotic therapy. All isolates were ciprofloxacin resistant. Bacteriologic and symptomatic UTI was detected in $4 \%$ vs. $6.6 \%$ and $4 \%$ vs. $3.9 \%$ in Group 1 and 2 respectively (p > $0.05)$.

\section{COMMENTS}

The use of needle guide for TPNB reduces technique variability; leading to a shorter learning curve for biopsy procedure; reduction in procedure time; and a consistent replicable procedure (9). However, the risk of cross-contamination is questionable. The disposable needle guide was introduced into the market in an effort to reduce the risk of infection.

The clinical infection rates after TRNP vary between 0.25 and $5 \%$ in past series, though other authors have reported rates of up to $7.5 \%(10,11)$. 
After biopsy incidence of bacteremia and bacteriuria were reported as $15 \%$ to $73 \%$ and $30 \%$ to $53 \%$ respectively $(12,13)$. Lindert et al. reported that bacteremia and bacteriuria after TPNB are common but usually asymptomatic (4). Urine cultures alone may not be useful for diagnosing infection (14). There is no unifying definition of infectious complications after TPNB. However, bacteriologic investigation (bacteriuria [more than $10^{5} \mathrm{CFU} / \mathrm{mL}$ ] versus no bacteriuria and bacteremia) and clinical diagnosis (no clinical symptoms of UTI versus clinical signs of UTI) at follow-up are determinant factors to evaluate the complications after TPNB. The clinical importance of positive urine culture in asymptomatic patients is unclear but one asymptomatic patient in group 2 required hospitalization due to ESBL producing E. Coli harvested in urine culture.

Bacteriologic UTI rate was found to be statistically similar in both groups. (4\% vs. $6.6 \%$ ).

Hospitalization was required in $4 \%, 6.6 \%$ in Group 1 and Group 2 relatively. The failures of quinolone prophylaxis might be the reason. Worldwide, quinolone-resistance rates among gram-negative bacilli are rising rapidly. Turkish Urinary Tract Infection Study Group recommended that fluoroquinolonesparing agents should be evaluated as alternative therapies by further clinical efficacy and safety studies (15). Ozden et al. reported the high incidence of ESBL producing E Coli after TRNP (16). New preventive protocols might be necessary for TPNB.

Obek et al. reported periprostatic lidocaine infiltration may be associated with a higher risk for infection due to the extra 2 punctures of lidocaine injection into the periprostatic area through the rectum, which is known to be highly colonized by bacteria (17). Intracapsular punctures during anesthesia can also contribute to the increased rate of infection after biopsy but both groups received the same type of periprostatic anesthesia in this study.

There are many factors that are related to the risk of infectious complications in prostate biopsies, such as lack of standardization among urologists in pre-biopsy antimicrobial prophylaxis, high incidence of resistant organisms, and the different methods of disinfection for reusable guides. Contamination of the tools used in biopsies has been reported to be the possible cause of infections occurring after TRUS-guided prostate biopsies (18). These devices have a needle guide that directs biopsy needle insertion. The biopsy needle repeatedly traverses the guide, affording the opportunity for fecal and blood materials to accumulate. Bioburdens can also accumulate in other parts of the transducer assembly. Brushes are required to clean the transducer assemblies to reduce the bioburden and remove proteins. Transducer assembly kits have not always been supplied with brushes. Not using brushes for cleaning has resulted in improperly reprocessed transducer assemblies, which could lead to pathogen transmission between patients. Gillespie et al. (5) detected an outbreak of Pseudomonas aeruginosa after prostate biopsy due to the insufficient manual cleaning of reusable needle guides. They concluded that clinicians performing TRUS-guided prostate biopsy procedures should follow the manufacturer's needle guide reprocessing recommendations or use disposable needle guides. In this study we used the Toshiba Fabio reusable needle guide. According to our results, all types of infectious complications such as asymptomatic bacteriuria, acute urinary infection, high fever, and bacteremia occurred at a statistically similar rate in both groups.

The permanent reusable needle guide, which has a long, narrow lumen may not have allowed adequate sterilization, as mentioned in previous studies, and may be the basis for the increased risk of infectious complications. It is essential to review the manufacturer's reprocessing recommendations and to establish appropriate procedures to avoid potential transmission of pathogens and subsequent patient concerns.

Potential sterilization problems with prostate biopsy equipment encourage the use of disposable equipment. Tuncel et al. (8) first reported that the use of a disposable needle guide reduces infectious complications. These authors determined that asymptomatic bacteriuria, high fever, and acute UTIs were statistically lower in the disposable group compared to the reusable group a $4.5 \%$ vs. $9 \% ; 5 \%$ vs. $10 \%$; and $2 \%$ vs. $9 \%$, respectively). Inadequate flushing of the lumen with disinfectant to eradicate microorganisms and the lack of availability of a device to clean the long, narrow lumen was suggested as the major problem with the use of a reusable needle guide for infectious complications. They concluded that the 
novel device which has been used as a disposable needle guide is a good alternative to reduce infectious complications. Our results are inconsistent with the results of the initial study but our study population was smaller than previous study populations, possibly affecting the power of our study. However, if the infectious complications occur due to the contamination of the needle guide, it may also occur due to the multiple reintroduction of the needle during the same biopsy. Using a reusable needle guide, even if the needle guide is changed after each pass of the needle, would provide little difference versus a disposable guide. The other explanation is that the physical features of the reusable needle guide in this study may have allowed proper sterilization. The separable and detachable features of the needle guide may be an important factor to prevent the possible infections. The permanent type of reusable needle guide which has been used in Tuncel et al. study (personal info from the author) might not have allowed the appropriate cleaning.

The core sampling notch was approximately $17 \mathrm{~mm}$ long by $1.2 \mathrm{~mm}$ in diameter. The procedure time including anesthesia application was 15 minutes for each case. There were no major technical problems during the procedure. However, the core length and the procedure time or some minor technical problems may individually affect the infection rate. We did not focus on this issue.

In our study, there were no statistically significant differences between the two groups regarding co-morbidity. Therefore, it is difficult to conclude that the risk of infection is less when using a disposable needle guide in patients with additional co-morbidities. Although the number of patients was limited in our study; a reusable needle guide was found to be safe in patients who had additional co-morbidities.

Reprocessing instructions for transrectal biopsy equipment, such as a transducer, biopsy channel bracket, and needle guide, is well-defined in the Food and Drug Administration recommendations (19). The risks of transmission of hepatitis B virus, hepatitis C virus, human immunodeficiency virus, during prostate biopsies were evaluated by Lessa et al. (20). Although, on aggregate, their findings do not provide evidence of disease transmission, although they do not rule out transmission in some cases. We did not focus on viral transmission in this study. The needle guide can be effectively disinfected with glutaraldehyde, but it must be disassembled from the transducer assembly before disinfection. We suggest that following the manufacturer's guidelines for the sterilization may be sufficient for using reusable instruments. The advantages of using a disposable needle guide are that it does not need to be reprocessed and it saves time, however, its cost is US\$ 5.00 per patient. Since the rate of infection appears to be same, whatever needle guide used, the issue of cost benefit ratio must always be kept in mind.

\section{CONCLUSION}

It seems to be ineffective to reduce the infection rate after the prostate biopsy by using a disposable needle guide. Large scale and randomized studies are necessary to determine the effect of disposable needle guide on infection rate after TPNB.

\section{ACKNOWLEDGEMENTS}

Mr. Rusen Celikoglu helped in this study.

\section{CONFLICT OF INTEREST}

None declared.

\section{REFERENCES}

1. Isen K, Küpeli B, Sinik Z, Sözen S, Bozkirli I: Antibiotic prophylaxis for transrectal biopsy of the prostate: a prospective randomized study of the prophylactic use of single dose oral fluoroquinolone versus trimethoprim-sulfamethoxazole. Int Urol Nephrol. 1999; 31: 491-5.

2. Raaijmakers R, Kirkels WJ, Roobol MJ, Wildhagen MF, Schrder FH: Complication rates and risk factors of 5802 transrectal ultrasound-guided sextant biopsies of the prostate within a population-based screening program. Urology. 2002; 60: 826-30.

3. Aron M, Rajeev TP, Gupta NP: Antibiotic prophylaxis for transrectal needle biopsy of the prostate: a randomized controlled study. BJU Int. 2000; 85: 682-5. 
4. Lindert KA, Kabalin JN, Terris MK: Bacteremia and bacteriuria after transrectal ultrasound guided prostate biopsy. J Urol. 2000; 164: 76-80.

5. Gillespie JL, Arnold KE, Noble-Wang J, Jensen B, Arduino M, Hageman J, et al.: Outbreak of Pseudomonas aeruginosa infections after transrectal ultrasoundguided prostate biopsy. Urology. 2007; 69: 912-4.

6. Huang YC, Ho DR, Wu CF, Shee JJ, Lin WY, Chen CS: Modified bowel preparation to reduce infection after prostate biopsy. Chang Gung Med J. 2006; 29: 395-400

7. Berger AP, Gozzi C, Steiner H, Frauscher F, Varkarakis $\mathrm{J}$, Rogatsch H, et al.: Complication rate of transrectal ultrasound guided prostate biopsy: a comparison among 3 protocols with 6,10 and 15 cores. J Urol. 2004; 171: 1478-80; discussion 1480-1.

8. Tuncel A, Aslan Y, Sezgin T, Aydin O, Tekdogan U, Atan A: Does disposable needle guide minimize infectious complications after transrectal prostate needle biopsy? Urology. 2008; 71: 1024-7; discussion 1027 8.

9. Phal PM, Brooks DM, Wolfe R: Sonographically guided biopsy of focal lesions: a comparison of freehand and probe-guided techniques using a phantom. AJR Am J Roentgenol. 2005; 184: 1652-6.

10. Griffith BC, Morey AF, Ali-Khan MM, Canby-Hagino E, Foley JP, Rozanski TA: Single dose levofloxacin prophylaxis for prostate biopsy in patients at low risk. J Urol. 2002; 168: 1021-3.

11. Peyromaure M, Ravery V, Messas A, Toublanc M, Boccon-Gibod L, Boccon-Gibod L: Pain and morbidity of an extensive prostate 10-biopsy protocol: a prospective study in 289 patients. J Urol. 2002; 167: 218-21.

12. Petteffi L, Toniazzo GP, Sander GB, Stein AC, Koff WJ: Efficiency of short and long term antimicrobial therapy in transrectal ultrasound-guided prostate biopsies. Int Braz J Urol. 2002; 28: 526-32.

13. Ruebush TK 2nd, McConville JH, Calia FM: A doubleblind study of trimethoprim-sulfamethoxazole prophy- laxis in patients having transrectal needle biopsy of the prostate. J Urol. 1979; 122: 492-4.

14. Zegarra Montes LZ, Sanchez Mejia AA, Loza Munarriz CA, Gutierrez EC: Semen and urine culture in the diagnosis of chronic bacterial prostatitis. Int Braz J Urol. 2008; 34: 30-7, discussion 38-40.

15. Yagci D, Yoruk F, Azap A, Memikoglu O: Prevalence and risk factors for selection of quinolone-resistant Escherichia coli strains in fecal flora of patients receiving quinolone therapy. Antimicrob Agents Chemother. 2009; 53: 1287-9.

16. Ozden E, Bostanci Y, Yakupoglu KY, Akdeniz E, Yilmaz AF, Tulek N, et al.: Incidence of acute prostatitis caused by extended-spectrum beta-lactamaseproducing Escherichia coli after transrectal prostate biopsy. Urology. 2009; 74: 119-23.

17. Obek C, Onal B, Ozkan B, Onder AU, Yalçin V, Solok $\mathrm{V}$ : Is periprostatic local anesthesia for transrectal ultrasound guided prostate biopsy associated with increased infectious or hemorrhagic complications? A prospective randomized trial. J Urol. 2002; 168: 558-61.

18. Centers for Disease Control and Prevention (CDC): Pseudomonas aeruginosa infections associated with transrectal ultrasound-guided prostate biopsies--Georgia, 2005. MMWR Morb Mortal Wkly Rep. 2006; 55: 776-7. Erratum in: MMWR Morb Mortal Wkly Rep. 2006; 55: 1177.

19. US Food and Drug Administration. FDA public health notification; Reprocessing of Reusable Ultrasound Transducer Assemblies Used for Biopsy Procedures. 2006. Available at http://www.fda.gov/MedicalDevices/Safety/AlertsandNotices/PublicHealthNotifications/UCM062086

20. Lessa F, Tak S, Devader SR, Goswami R, Anderson $\mathrm{M}$, Williams I, et al.: Risk of infections associated with improperly reprocessed transrectal ultrasound-guided prostate biopsy equipment. Infect Control Hosp Epidemiol. 2008; 29: 289-93.

\author{
Correspondence address: \\ Dr. Cenk Gurbuz \\ 2222 Medical District Dr. 4339 \\ Dallas, TX 75235, USA \\ Fax: + 146 9422-9910 \\ E-mail: gurbuzcenk@yahoo.com
}




\section{EDITORIAL COMMENT}

Transrectal guided prostate needle biopsy (TPNB) is one of the most common outpatient diagnostic procedures in urology clinics and in this study the authors analyzed whether the use of disposable needle guide decrease the infectious complication after TPNB. Fever after prostate biopsy was developed in 3.5\% patients (1). Urosepsis and bacteremia were rare but bothersome complications. Antibiotic prophylaxis including ciprofloxacin therapy has been administrated in most of the urology clinics to prevent infectious complications. Recently, Tuncel et al. suggested that the use of disposable needle guide could reduce the infection risk after prostate biopsy (2). However, in the current study the authors showed that it is ineffective to prevent infection.

Rutala et al. demonstrated that probe disinfection with glutaraldehyde greater than $2 \%$ concentration could be achieved when prostate needle guide was removed (3). Therefore, adequate cleaning and disinfection could explain the similar infection risks of use disposable and reusable needle guide. However, the small number of patients included in the study group prevents definite clinical judgment.
In spite of these controversially results, the disposable needle guide with a price of $\$ 5.00$ should be used for biopsy to overcome the difficulty in standardization of proper disinfection procedures in urology clinics. Whenever disposable needle is not available, mechanical cleaning to remove biologic material followed by 20 minutes of disinfection with glutaraldehyde should be performed after removing the reusable needle guide.

\section{REFERENCES}

1. Raaijmakers R, Kirkels WJ, Roobol MJ, Wildhagen MF, Schrder FH: Complication rates and risk factors of 5802 transrectal ultrasound-guided sextant biopsies of the prostate within a population-based screening program. Urology. 2002; 60: 826-30.

2. Tuncel A, Aslan Y, Sezgin T, Aydin O, Tekdogan U, Atan A: Does disposable needle guide minimize infectious complications after transrectal prostate needle biopsy? Urology. 2008; 71: 1024-7; discussion 10278.

3. Rutala WA, Gergen MF, Weber DJ: Disinfection of a probe used in ultrasound-guided prostate biopsy. Infect Control Hosp Epidemiol. 2007; 28: 916-9.

\section{Dr. Onur Kaygýsýz. Yalova State Hospital Department of Urology Yalova, TURKEY E-mail: onurkygsz@yahoo.com}

\section{EDITORIAL COMMENT}

The authors should be commended on such a novel concept to potentially reduce infection after transrectal prostate needle biopsy. The methodology used to detect presence of infection was comprehensive and well designed. The randomized cohorts did not demonstrate a significant difference with regard to infection rates as a function of disposable versus reusable needle guide. The bacteriologic infection rate was $4 \%$ in both groups while surprisingly, the symptomatic urinary tract infection rate was greater for the disposable needle guide.

Infection rates after transrectal biopsy are not negligible and certainly, any effort to reduce them should be explored. Our group has offered stereotactic 
transperineal prostate biopsy (STPB) for patients that have had prior negative transrectal prostate biopsy despite continued elevation of PSA $(1,2)$. While $40 \%$ of these patients are identified as having occult malignancy after STPB, an additional benefit has been a true null of post procedure infection. I acknowledge that STPB is not widely accepted as a first line biopsy technique, but given the high diagnostic yield and negligible risk of infection, STPB may be more commonplace in the future.

\section{REFERENCES}

1. Moran BJ, Braccioforte MH: Stereotactic transperineal prostate biopsy. Urology. 2009; 73: 386-8.

2. Moran BJ, Braccioforte MH, Conterato DJ: Rebiopsy of the prostate using a stereotactic transperineal technique. J Urol. 2006; 176: 1376-81; discussion 1381.

Dr. Brian J. Moran Chicago Prostate Cancer Center Westmont, Illinois, USA E-mail: seeds@prostateimplant.com 